Forensic Science International: Genetics 2019; 43:102153: 1-6

(DOI: 10.1016/j.fsigen.2019.102153)

\title{
A retrospective study on the transfer, persistence and recovery of sperm and epithelial cells in samples collected in sexual assault casework
}

\author{
Ane Elida Fonneløp ${ }^{a}$, Helen Johannessen ${ }^{a}$, Guro Heen $^{\mathrm{a}}$, Karen Helene Molland ${ }^{\mathrm{a}}$; Peter Gilla,b
}

${ }^{a}$ Oslo University Hospital, Norway, and ${ }^{b}$ University of Oslo, Oslo, Norway

\section{Introduction}

In rape cases the detection of spermatozoa in a sample from an intimate swab is frequently used as evidence of sexual activity. Given ejaculation and normal sperm quality, it has been shown that spermatozoa will normally be detected in intimate samples with a time since intercourse (TSI) up to 48 hours, and in some cases up to 6 days $[1,2]$. However, in a large proportion of casework, no spermatozoa are detected [3-5]. This could be because a condom was used, the assaulter did not ejaculate or is sterile, the sampling was insufficient, or sperm cells were already degraded by the time of sampling. In these cases there could be a higher chance of detecting female epithelial vaginal cells on the perpetrator's penis if sampling has taken place within 24 hours [6-8]; persistence of more than 24 hours has not been tested in a controlled experiment to our knowledge. There is also a possibility of detecting male DNA from epithelial cells shed from the penis, hands or from saliva, and deposited during contact with other parts of the body e.g. grabbing of the neck, breasts, and kissing or biting the victim's skin. These areas can be sampled to look for perpetrator's DNA. Saliva is considered a good source of DNA; controlled experiments have demonstrated persistence of up to 96 hours for saliva deposited on skin [9]. In contrast skin cells are reckoned to be a poor source of DNA; it has been shown that DNA from skin contacts will be detected less frequently, even directly after contact, and will be "removed" more rapidly from another person's body [10]. The persistence and detection of epithelial cells deposited on the body will be affected by factors such as new contacts, activity and personal hygiene, e.g. bathroom visits or showering. A DNA sample from a vaginal swab will be dominated by the female mucosal cells and often no male DNA will be detected with the standard autosomal analysis. In some cases, if the quantitation results reveal that a small amount of male DNA is present, Y-STR analysis that targets the male DNA on the Y chromosome can be used. However, rapid degradation of epithelial cells is expected in addition to mechanical removal.

In Norway, collection of biological evidence from a victim of sexual assault is carried out by a specialized medical rape unit or a doctor. A collection kit for sexual assault cases has been developed: including sterile cotton swabs (Puritan), water tubes, pre-labelled paper bags (tick off box record for common areas of sampling), unlabelled paper bag, a comb and an examination form with questions to the victim and instructions to the examiner on how to carry out the sampling of biological material. The doctors are encouraged to do full anamneses as new information may be 
added to the case at a later state. In general, samples from genitals and finger nails are collected; samples from skin areas are taken if the victim describes a specific contact.

The suspect, on the other hand, is usually examined by a doctor or a scene of crime officer in the arrest with standard sampling equipment (sterile cotton swabs (Puritan), water tubes and paper bags).

If the case is further investigated the police may request The Oslo University Hospital, Section of Forensic Biology, to analyse the biological samples collected from the victim and/or the suspect. Typically, all the samples from the medical examination are included with the request form, and the forensic scientist has to prioritise which samples are examined based on case information such as relation between victim and offender, type of activity/sexual offence, activity after the offence (shower etc.) and time between alleged assault and sampling. Based on the information and knowledge of transfer and persistence of biological material, the scientist will choose the samples that will possibly provide useful information to the case and are likely to detect semen or epithelial cells from the involved persons.

In this paper we present data from a retrospective study from sexual assault cases, analysed in the period 2013-2015 at the section, where all standard analyses have been conducted using the methods with increased sensitivity amplification using the PowerPlex ${ }^{\circledR}$ ESX $17 /$ ESX 17 Fast and separation on the Applied Biosystems 3500xl Genetic analyser. In this study "positive findings" refer to evidence to support the proposition that the DNA profile was contributed by the person of interest (POI) and do not just correspond to detection of cell type, i.e. sperm cells. Thus, the positive findings are case relevant. Transfer and persistence data of epithelial cells detected on the victim or suspects' skin areas are also included. There are controlled experiments published on recovery of epithelial cells after skin contact [10], but many uncontrolled factors will influence the persistence and detection in "real life" (casework). Consequently, these data may be more representative of the expectations of the findings and can serve as a guide to help prioritize samples collected at different times since an assault, In addition, expectations of "success rates" can be used to address questions related to positive or negative results.

\section{Methods}

\subsection{Data collection and classification}

Data were collected from cases of sexual assault analysed at the institute during the period 20132015. A total of 1499 cases, were reviewed to study persistence of cells deposited on the body. Samples collected from either the suspect or the victim's body, and with a known time between alleged assault and sampling were included. In some cases a time interval is given instead of a specific time of assault or sampling (e.g. victim does not remember the exact time, or the assault lasted for a longer period), we only included cases with a maximum 12 hours interval in this study. Cases where only exhibits (e.g. clothing) were examined were excluded in this study.

Data were collected and divided into cell type, location of sampling, time between alleged assault and sampling and whether or not a DNA profile from the other part (victim or suspect/accused) was obtained with autosomal and/or Y STR analysis. Detection of spermatozoa was recorded based on detection by microscopy. In summary, approx. $1 / 3$ of the swab tip was sampled and incubated with 
$50 \mu \mathrm{l}$ Milli-Q water at room temperature in a shaker $(600 \mathrm{rpm})$ for 60 minutes. Subsequently $3 \mu \mathrm{l}$ were added in two parallels to designated zones on a microscope slide, dried and stained with Christmas three staining. Microscopic confirmation of sperm cells was classified into three categories dependent on the highest number of sperm cells detected in one parallel: 1) 3-19 sperm cells, 2) 2099 sperm cells and 3 ) $\geq 100$ sperm cells. Slides with less than three sperm cells were regarded as negative (and reported as absence of sperm cells). The presence/absence of epithelial cells during the microscopy examination was also recorded, but not further specified in this data set. Presumptive test for semen (Acid phosphatase test, and occasionally Seratec ${ }^{\circledR}$ PSA semiquant test kit and/or RSID semen test (Independents Forensics)) are routinely carried out when examining for semen, but only the confirmation test, i. e. detection of sperm cells by microscopy, are included in this data set. Saliva examinations were carried out, if requested, using the Phadebas Press test and occasionally RSID saliva test (Independents Forensics). The data from these presumptive tests are included in the data set for skin samples.

Data were recorded with regards to sperm or epithelial cells and further divided into the following location categories; Internal vaginal swabs, external genital swabs (exterior vagina and anus), internal rectal swabs, oral swabs, hand swabs (fingernails, fingertips and palm), skin swabs and penile swabs. A maximum of one sample in each cell category (epithelial or sperm) was included from each location in this study.

\subsection{DNA analysis}

Casework samples were extracted by the $5 \%$ Chelex $^{\circledast}$ procedure from Bio-Rad (epithelial samples) or QIAamp ${ }^{\circ}$ DNA Microkit from Qiagen (differential extraction of semen samples) according to current practice at the department. All samples were quantified with Quantifiler ${ }^{\circledR}$ Duo Kit or Quantifiler ${ }^{\circledR}$ Trio kit (both Applied Biosystems ${ }^{\circledR}$ ) on the 7500 Real-Time PCR system (Applied Biosystems ${ }^{\circledR}$ ).

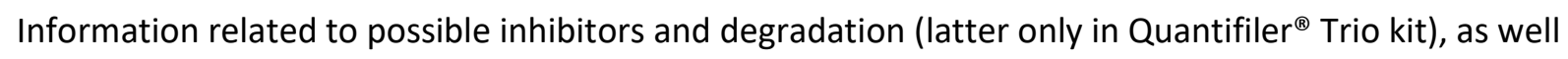
as the total human / male DNA ratio in a sample, affect the analysis strategy (for instance, purification step, second analysis with a complementary kit or a Y-STR analysis respectively), but these parameters were not collected, thus not further studied in this data set. All samples were amplified using the PowerPlex ${ }^{\circledR}$ ESX 17 / PowerPlex ${ }^{\circledR}$ ESX 17 Fast System kit (Promega) as recommended by the manufacturer ( $0.5 \mathrm{ng}$ template, $25 \mu \mathrm{L}$ reaction volume and 30 amplification cycles). Some of the samples were also analyses with Yfiler ${ }^{\mathrm{TM}}$ PCR Amplification Kit or Yfiler ${ }^{\mathrm{TM}}$ Plus PCR Amplification Kit (Thermo Fisher) as recommended by the manufacturer (1 ng template, $25 \mu \mathrm{L}$ reaction volume and 30 amplification cycles). Samples that had lower concentrations than the recommended template amount were amplified with the maximum template volume of $17.5 \mu \mathrm{L}$ (ESX 17 Fast) or $10 \mu \mathrm{L}$ (Yfiler/ Yfiler Plus), but samples with a lower concentration than $0.004 \mathrm{ng} / \mu$ l were not amplified. Amplification was carried out using a GeneAmp ${ }^{\circledR}$ PCR System 9700 (Applied Biosystems $\left.{ }^{\circledR}\right)$. Samples were injected on the Applied Biosystems 3500xl Genetic Analyzer at $1.2 \mathrm{kV}$ for 10s (ESX 17), 24s (ESX 17 Fast) and 12s (Yfiler Plus), The results were analysed using the GeneMapper ${ }^{\circledR}$ ID-X Software (Applied Biosystems ${ }^{\circledR}$ ) and the limit of detection (LOD) for alleles was set to 200 RFU (ESX 17 / ESX 17 Fast) and 100 RFU (Yfiler Plus). The samples analysed with Yfiler were injected on the Applied Biosystems 3130 Genetic Analyzer and the LOD was set to 50 RFU.

The stochastic threshold was set to $1200 \mathrm{RFU}$. The method used to assign contributors to a DNA profile was based on either matching a single source full profile or analysis of their respective mixture proportions in a sample as described by Gill et al. [11]. Good quality 2 or 3 person mixtures, i.e. all 
alleles from the contributors are considered detected, are suitable for further comparison with reference samples, while mixtures of more than 3 contributors were only considered if there was a clear major contributor. For weaker profiles consisting of 2 or 3 persons, where allele drop out is expected, only the major contributor(s) was reported. If the minor contributor was a partial match to the POI, with exception of a few alleles which could be explained by allele drop out, the POI was reported as not excluded as a contributor to the sample. Likelihood ratio (LR) calculations were carried out in many of the cases but are not normally reported in the statement.

\section{Results}

The final dataset consist of 2349 samples from 766 cases; 222, 267 and 277 cases from 2013, 2014 and 2015 respectively. From the original 1499 cases 325 cases were excluded because information about the time of the incidence and/or medical examination were missing or the given time intervals were too large (more than 12 hours), hence these data could not provide useful information about persistence. In addition 370 cases were excluded since only exhibits (e.g. clothing) were examined. Finally 38 cases were also excluded due to missing information.

The number of sampled locations analysed in each case varied between 1 and 9, table 1 . At least one positive sample (transfer of cells between suspect and victim) was detected in 356 (47\%) of the cases.

Table 1: Number of cases including 1-9 sampled locations and the average frequency of positive samples

\begin{tabular}{|c|c|c|}
\hline Number of locations & cases & Freq. of positive \\
\hline 1 & 132 & 0.41 \\
\hline 2 & 184 & 0.41 \\
\hline 3 & 176 & 0.20 \\
\hline 4 & 139 & 0.23 \\
\hline 5 & 81 & 0.24 \\
\hline 6 & 30 & 0.23 \\
\hline 7 & 14 & 0.21 \\
\hline 8 & 8 & 0.17 \\
\hline 9 & 2 & 0.56 \\
\hline
\end{tabular}

\subsection{Results from analysis of spermatozoa}

\section{A total of 1223 samples, in 627 cases, examined for detection of sperm cells (microscopy), are} included in the dataset. A positive sample is defined as detection of spermatozoa, where the DNA result of the sperm fraction supported the prosecution's proposition that the POI contributed to the sample. At least one positive sample was detected in 194 cases (31\%). The samples in the data set were collected between 1 and 144 hours after the alleged assault occurred, with the majority collected within 24 hours, (fig. 1). Positive results were detected in samples collected up to 72 hours after deposition. 


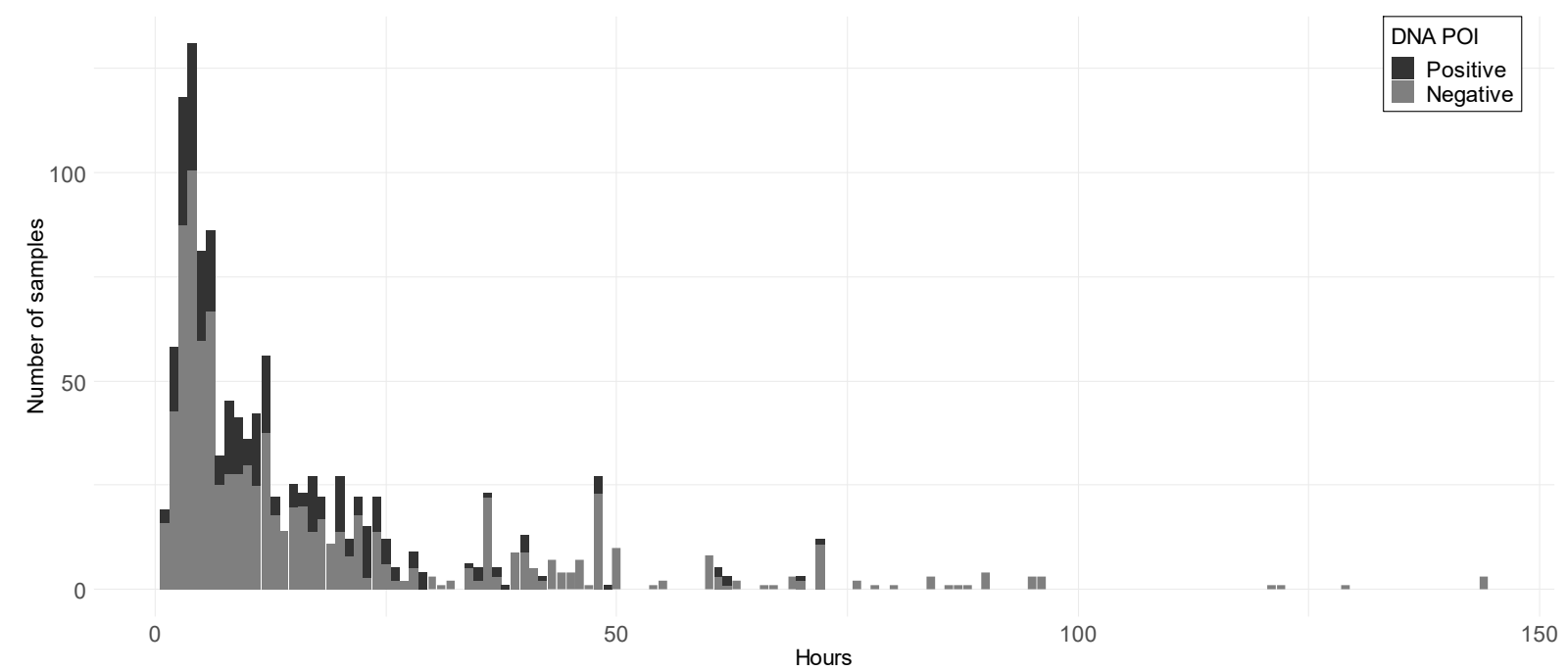

Fig. 1 Bar graph displaying the number of positive and negative spermatozoa samples according to the time between incidence and sampling

154

155

156

157

158

159

160

161

162

163

\section{4}

The percentage of positive samples decreased with increasing time between assault and sampling (table 2). The percentage difference between positive samples at different time intervals (table 2) was significant between at least two of the groups (Pearson's Chi-squared, $p=0.001$ ). In oral swabs positive samples were obtained up to 12 hours after the alleged assault, while the 12 samples collected at 13-24 hours were all negative. Positive samples could be detected up to 35 hours in rectal swabs, 72 hours in internal vaginal swabs and 62 hours in external genital swabs.

Table 2: Percentage positive samples (total number of samples) analysed for detection of spermatozoa divided into location of sampling (internal vaginal swabs, external vaginal swabs, rectal swabs, oral swabs and skin surface) and in 4 categories according to the time between incidence and sampling.

\begin{tabular}{|c|c|c|c|c|}
\hline Sample & $\mathbf{1}$ & $\mathbf{2}$ & $\mathbf{3}$ & $\mathbf{4}$ \\
$\mathbf{1 - 2 4 h}$ & $\mathbf{2 5 - 4 8 h}$ & $\mathbf{4 9 - 7 3 h}$ & $\mathbf{7 4 h}$ \\
\hline $\begin{array}{c}\text { Internal vaginal } \\
\text { swabs }\end{array}$ & $30 \%(413)$ & $24 \%(82)$ & $17 \%(29)$ & $0(18)$ \\
\hline $\begin{array}{c}\text { External genital } \\
\text { swabs }\end{array}$ & $26 \%(406)$ & $24 \%(55)$ & $13 \%(15)$ & $0(4)$ \\
\hline Rectal swabs & $19 \%(68)$ & $5 \%(19)$ & $0(7)$ & $0(4)$ \\
\hline Oral swabs & $11 \%(71)$ & $0(2)$ & $0(1)$ & - \\
\hline Skin surface & $62 \%(29)$ & - & - & - \\
\hline
\end{tabular}

The samples referred to as negative are mainly due to no detection of spermatozoa (768). However in some of the samples (94) sperm cells were detected, but no DNA result was obtained. The majority of these samples (88) was classified as category 1 during microscopy (3-19 sperm cells), while a few (6) were classified as category 2 (20-99 sperm cells), most of these samples were collected within 48 hours (table 3). 
Table 3: Observed spermatozoa categories for samples with no DNA results divided in to four categories of time between incidence and sampling.

\begin{tabular}{|c|c|c|c|c|}
\hline Spermatozoa & $\begin{array}{c}\mathbf{1} \\
\mathbf{1 - 2 4 h}\end{array}$ & $\begin{array}{c}\mathbf{2} \\
\mathbf{2 5 - 4 8 h}\end{array}$ & $\begin{array}{c}\mathbf{3} \\
\mathbf{4 9 - 7 3 h}\end{array}$ & $\begin{array}{c}\mathbf{4} \\
\mathbf{7 4 \geq h}\end{array}$ \\
\hline 1 (3-19 sperm cells) & 68 & 14 & 5 & 1 \\
\hline 2 (20-99 sperm cells) & 4 & 1 & 0 & 1 \\
\hline Negative & 599 & 106 & 39 & 24 \\
\hline
\end{tabular}

\section{Excluded samples}

For 38 samples from 28 cases spermatozoa was detected but the DNA profile did not match the suspect, the DNA profile from an individual unrelated to the crime (e.g. boyfriend). These samples were excluded from the dataset.

\subsection{Results from analyses of epithelial cells}

The dataset includes analysis of 1126 epithelial samples from 568 cases. Positive results that supported the prosecution proposition in the specific case that the POI contributed to the sample were obtained in 218 cases (38\%). Persistence of cells was shown up to 72 hours post contact, however, the majority of positive samples were collected in the period 1-12 hours, fig. 2.

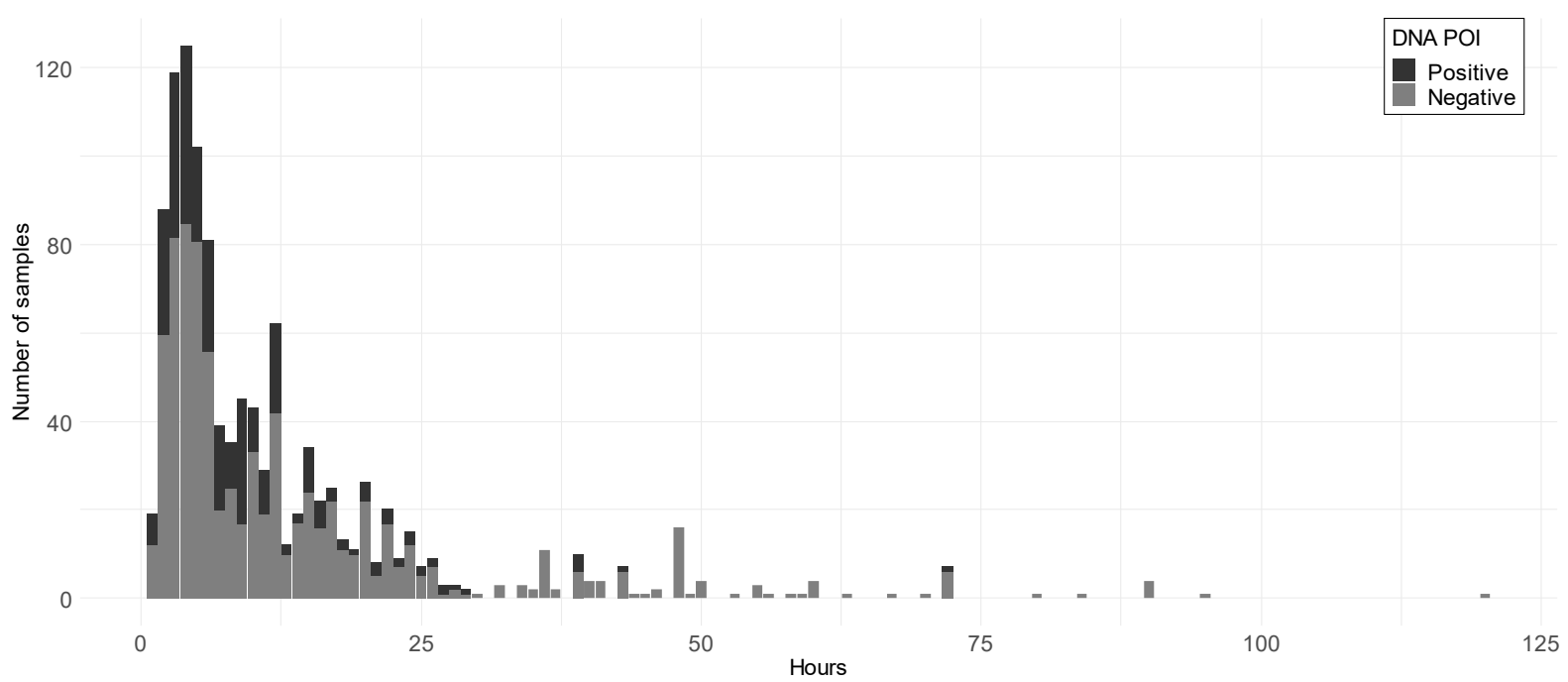

Fig. 2 Bar graph displaying the number of positive and negative results of epithelial samples according to the time between incidence and sampling

Percentage of positive samples, where the DNA evidence supported the prosecution proposition, decreased when time between incidence and sampling increased, table 4. The percentage differences between positive samples at different time intervals (table 4) were significant between at least two time groups (Pearson's Chi-squared, $\mathrm{p}<<0.001$ ). Positive samples were detected up to 12 hours in external genital swabs, 27 hours in hand swabs, 43 hours in skin swabs, 39 hours in penile swabs and the longest persistence time was observed in a sample from the internal vagina which was collected 72 hours after the incidence (14/16 alleles, Y-filer). Only few samples have been analysed 
when the time between assault and sampling exceeds 49 hours, hence all samples exceeding this time has been combined in class 5 in table 4 . Only few samples have been collected from the internal vagina, rectum and mouth for epithelial analysis: five of these samples provided a positive result, all from internal vaginal swabs and all based on $Y$ chromosome analysis where four of the samples were collected within 12 hours.

Table 4: Percentage positive samples (total number of samples) analysed for detection of epithelial cells divided into location of sampling internal vaginal swabs, external vaginal swabs, rectal swabs, oral swabs, hand swabs, skin swabs and penile swabs) and in 5 categories according to the time between incidence and sampling.

\begin{tabular}{|c|c|c|c|c|c|}
\hline Sample & $\begin{array}{c}\mathbf{1} \\
\mathbf{1 - 1 2 h}\end{array}$ & $\begin{array}{c}\mathbf{2} \\
\mathbf{1 3 - 2 4 h}\end{array}$ & $\begin{array}{c}\mathbf{3} \\
\mathbf{2 5 - 3 6 h}\end{array}$ & $\begin{array}{c}\mathbf{4} \\
\mathbf{3 7 - 4 8 h}\end{array}$ & $\begin{array}{c}\mathbf{5} \\
\mathbf{4 9}<-\end{array}$ \\
\hline $\begin{array}{c}\text { External genital } \\
\text { swabs }\end{array}$ & $14 \%(242)$ & $0(68)$ & $0(10)$ & $0(17)$ & $0(10)$ \\
\hline Hand swabs & $45 \%(108)$ & $38 \%(24)$ & $17 \%(6)$ & $0(5)$ & $0(6)$ \\
\hline Skin swabs & $30 \%(268)$ & $18 \%(67)$ & $30 \%(10)$ & $50 \%(8)$ & $0(2)$ \\
\hline Penile swabs & $59 \%(152)$ & $43 \%(47)$ & $24 \%(17)$ & $8 \%(12)$ & $0(12)$ \\
\hline Internal vaginal swabs & $50 \%(8)$ & $0(2)$ & $0(1)$ & $0(3)$ & $33 \%(3)$ \\
\hline Rectal swabs & $0(8)$ & $0(6)$ & - & $0(2)$ & $0(1)$ \\
\hline Oral swabs & $0(2)$ & - & - & - & - \\
\hline
\end{tabular}

Positive epithelial samples collected on the skin was further studied to look for differences is success rates between different locations. There was a significant difference between at least two groups (Pearson's Chi-squared, $p=0.001$ ), for instance positive results were obtained in $41 \%$ of the samples collected from the breast/chest area while $15 \%$ were positive from lips and around mouth, table 5 . The longest persistence time observed was a positive sample collected from breast/chest area 43 hours post assault. A selection of the skin samples (94) were tested for the presence of saliva as explained in section 2.1. In the group of samples that tested positive for saliva $64 \%$ had a positive DNA result consisted to be from the POI, while among the samples that tested negative $27 \%$ of the samples gave positive DNA results, table 6. 


\begin{tabular}{|l|c|c|}
\hline Skin locations & Total & Positive (\%) \\
\hline Face & 45 & 38 \\
\hline Lips and around mouth & 62 & 15 \\
\hline Neck/troath & 92 & 33 \\
\hline Breast/Chest & 73 & 41 \\
\hline Arm & 27 & 11 \\
\hline Legs & 5 & 40 \\
\hline Seat & 12 & 8 \\
\hline Thigh & 20 & 10 \\
\hline Body (rest) & 19 & 21 \\
\hline
\end{tabular}

Table 6: Number of samples with a positive and negative result when tested for the presence of saliva ( $\alpha$-amylase) and the percentage that provided a profile from the POI

\begin{tabular}{|l|c|c|}
\hline & Number of samples & Positive (\%) \\
\hline Saliva $\div$ & 59 & 27 \\
\hline Saliva + & 33 & 64 \\
\hline
\end{tabular}

\section{Discussion}

The positive findings in this study correspond to the detection of DNA where the strength of the evidence supported the prosecution proposition that it could be attributed to the POI. The results reflect real case data, rather than controlled experiments, therefore there is always some uncertainty about whether case circumstances etc. are correct. Nevertheless, such data provide a new perspective on transfer and persistence of DNA that cannot be achieved with controlled experiments. Hence this study differs from other retrospective studies where only the presence of semen in samples was studied [3,4]. Positive results were detected in samples collected up to 72 hours after deposition. For two samples spermatozoa were detected after this point ( 80 and $96 \mathrm{~h}$ ), but no DNA profiles were obtained. The data supports the findings by Casey et al. [3] which concluded that the chance of detecting sperm in vaginal swabs are highly reduced beyond a time since intercourse (TSI) of 72 hours. Several papers present detection of sperm cells up to 5 to 7 days after deposition $[1,3,4,12]$, some of the difference observed may be due to difference in preparation of samples for microscopy (e.g. sampling the entire swab vs. one third) [4]. However, our data does not contain positive DNA-results from sperm when the TSI exceeded 3 days.

We observed 38 samples from 28 cases where spermatozoa were detected, but the subsequent DNA analyses showed that it had an origin from an individual unrelated to the crime (e.g. boyfriend). As these cells were most likely deposited at a time different from the alleged assault, such samples could bias persistence data and they were excluded. In 94 samples spermatozoa were detected but no DNA profiles were obtained. The reason why no DNA profile was achieved could be because of too few sperm cells present in samples and loss of cells during the differential extraction, which is a common event in samples where donor cells are in excess [13]. As we do not have any DNA profiling 
results in these cases, we cannot be certain that these cells are case related. Hence, the inclusion of these samples in the data set could potentially bias persistence rates.

Most of the samples included in our dataset are collected within 24 hours after the alleged offence. There is an expectation to detect sperm cells from these samples if an ejaculation with normal sperm quality has occurred [1, 2]. Still, in $70 \%$ of the samples collected within 24 hours, no sperm from the assaulter/suspect was detected. In many of the sexual assault cases the victim is under influence of alcohol or drugs, or sleeping, and not able to recall or notice details of the assumed sexual activity. In these cases it is possible that the high degree of negative findings can be explained by no sexual activity, no ejaculation or use of a condom. This illustrates the importance of including additional samplings such as skin, hands or penile swabs, if the time since the alleged assault is within the detection limit.

In rectal swabs, sperm that provided a DNA profile, providing evidence to support the prosecution proposition that it came from the suspect, was detected up to a TSI of 35 hours, however there was only one positive observation beyond 24 hours. Casey et al. [3] also reported the majority of sperm positive swabs in samples collected within 48 hours. The oral swabs were usually negative, and the data collected over the 3 years ( 73 samples) confirmed just 8 incidents of positive findings. In all these cases the TSI was within 12 hours, which is in line with the findings of Willot and Crosse [14] who detected sperm cells in oral swabs up to a TSI of 8 and in saliva samples for up to 13 hours. It also correspond with findings by Casey et al. [3] who observed a low expectation of detecting sperm cells up to 15 hours. Detection of spermatozoa on the skin surface within 24 hours (no samples were collected after $24 \mathrm{~h}$ ) has the highest success rate (62\%). The probable explanation of this high rate is that these samples are collected and analysed if the victim explains ejaculation on this area specifically and has not showered before examination.

If no spermatozoa were detected in the vaginal swabs, the external vaginal/anal swabs were usually analysed for the presence of epithelial cells from the perpetrator. However, the data showed that there is a small chance of detecting case relevant (POI) epithelial cells in these samples, especially when the time since contact increases. Only 34 of the 346 samples in the dataset provided a DNA profile where the strength of the evidence supported the prosecution proposition that it came from the suspect. All positive samples were collected within 12 hours after the alleged assault, suggesting that external genital samples collected beyond a TSI of 24 hours, should not be examined for epithelial cells routinely. Similar findings were observed in a controlled experiment on persistence of skin cells deposited on skin [10], where there was a significant decrease in detection when three hours had past and only one incidence of detection of DNA from the depositor after 24 hours.

The longest persistence of epithelial cells was detected on skin and penile swabs. Almost $50 \%$ of the penile swabs provided DNA profiles where the strength of the evidence supported the prosecution proposition that it came from the victim, the majority of these were sampled within 24 hours, but also up to a TSI of 39 hours. The success rates differ from the observations by Kaarstad et al. [8] who observed a detection of a female DNA profile in $27 \%$ of cases; however, they did not divide success rates into classes by time between assault and sampling, although they highlighted that the majority of positive samples were collected between 1-15 hours. In addition, some of the differences in success rate could be explained by more sensitive analysis methods used in the present study. In a controlled experiment on presence of female DNA on post coital penile swabs collected between 1 
and 24 hours after intercourse, China et al. [6] observed that female DNA could be detected in all samples but that the amount of female DNA decreased with time. Corresponding observations were also observed in a similar study by Farmen et al. [7]. To our knowledge there are no published controlled studies that measures persistence of female cells on the penis beyond 24 hours. It is however expected that if the cells dry and are undisturbed, these cells can persist for several days, as previously demonstrated for saliva on skin [9] which agrees with the persistence of up to 36 hours detected in this study. It is likely that mechanical removal e.g. contact with clothing, bathroom visits and showering will occur over time, knowledge about this type of activity is often not available in casework. The data show the importance of collecting penile swabs in cases where no spermatozoa are detected in intimate samples from the victim. Consequently, police should prioritize collecting these samples to a higher degree than is current practice (penile swabs were analysed in less than $30 \%$ of all cases included in this study).

Only few samples have been collected from the internal vagina, rectum and mouth for epithelial analysis. There is a low expectancy of detecting epithelial cells from the POI in these locations as the samples are likely to be dominated by mucosa cells from the donor itself. There are however five positive samples in this class, all from internal vaginal swabs and all based on $Y$ chromosome analysis. The majority of these samples were collected only few hours post the accused assault, while one sample was collected 72 hours post. McDonald et al. [15] observed occasions of persistence of Y-STR profile with 10 or more alleles in cases with no detected spermatozoa up to 48 hours, no samples beyond this time was included in their study.

If the victim and the suspect have had undisputed recent social contact prior to the alleged offence, samples that cannot provide useful additional information, e.g. fingernail scraping, are not examined. Nevertheless, positive results were obtained from skin swabs up to 43 hours after the incident, though not many samples beyond 24 hours were examined. It is possible that the positive results beyond 24 hours can be explained by the presence of mucosal epithelial cells. In contrast to the shorter persistence demonstrated for skin cells deposited on skin [10], persistence of saliva on skin has been demonstrated up to 96 hours [9]. Two of the four positive samples collected from skin beyond 37 hours after contact were $\alpha$-amylase positive; this is however only a presumptive test. The $\alpha$-amylase activity can also be reduced over time; hence a positive test may not be achieved although saliva is present. Hand swabs, collected from both victim and suspect, provided persistence data up to 36 hours post contact, though only a few samples were examined beyond 1 day. Again we hypothesised that the source of the cells persisting more than 12 hours could be mucosa cells as skin cells have previously been shown to diminish quite rapidly from hands [16, 17] or from fingernails [18] , compared to vaginal mucosal cells [19]. Nevertheless, the casework data indicate that there is a very small chance of epithelial persistence and recovery of a profile on hands, skin and penis after a TSI of 48 hours.

\section{Conclusion}

This study presents transfer and persistence data of sperm and epithelial cells in samples collected from the victim or suspect's body in sexual assault cases. The positive findings refer to evidence to support the proposition that the DNA profile was contributed by the POI, thus the positive findings are case relevant. Sperm cells had the highest persistence rate in internal vaginal swabs, and were detected up to 72 hours post assault, but the majority of the positive samples were collected within 
48 hours. Skin and penile swabs demonstrated persistence of epithelial cells up to 48 hours, the majority of the positive samples were within 24 hours. In external genital swabs persistence of epithelial cells were not detected if collection of the sampling occurred beyond 12 hours post assault. The data set provided in this study may serve as a guide in what samples to prioritize for analysis dependent on time between assault and medical examination of the victim or suspect, and furthermore displays the expectancy of findings when questions in regards to positive or negative results are addressed in court.

\section{References}

1. Davies, A. and E. Wilson, The persistence of seminal constituents in the human vagina. Forensic science, 1974. 3: p. 45-55.

2. Astrup, B.S., et al., Detection of spermatozoa following consensual sexual intercourse. Forensic science international, 2012. 221(1-3): p. 137-141.

3. Casey, D.G., et al., The persistence of sperm and the development of time since intercourse (TSI) guidelines in sexual assault cases at forensic science Ireland, Dublin, Ireland. Journal of forensic sciences, 2017. 62(3): p. 585-592.

4. Willott, G. and J. Allard, Spermatozoa-their persistence after sexual intercourse. Forensic science international, 1982. 19(2): p. 135-154.

5. Hellerud, B.B., et al., Semen detection: A retrospective overview from 2010. Forensic Science International: Genetics Supplement Series, 2011. 3(1): p. e391-e392.

6. Cina, S.J., et al., Isolation and identification of female DNA on postcoital penile swabs. The American journal of forensic medicine and pathology, 2000. 21(2): p. 97-100.

7. Farmen, R.K.B., et al., Assessing the presence of female DNA on post-coital penile swabs: Relevance to the investigation of sexual assault. Journal of forensic and legal medicine, 2012. 19(7): p. 386-389.

8. Kaarstad, K., et al., The detection of female DNA from the penis in sexual assault cases. Journal of forensic and legal medicine, 2007. 14(3): p. 159-160.

9. Kenna, J., et al., The recovery and persistence of salivary DNA on human skin. Journal of Forensic Sciences, 2011. 56(1): p. 170-175.

10. Bowman, Z.E., et al., Detection of offender DNA following skin-to-skin contact with a victim. Forensic science international: genetics, 2018. 37: p. 252-259.

11. Gill, P., et al., DNA commission of the International Society of Forensic Genetics: Recommendations on the interpretation of mixtures. Forensic Science International, 2006. 160(2-3): p. 90-101.

12. Allard, J., The collection of data from findings in cases of sexual assault and the significance of spermatozoa on vaginal, anal and oral swabs. Science and Justice, 1997. 37(2): p. 99-108.

13. Vuichard, S., et al., Differential DNA extraction of challenging simulated sexual-assault samples: a Swiss collaborative study. 2011. 2(1): p. 11.

14. Willott, G. and M. Crosse, The detection of spermatozoa in the mouth. Journal of the Forensic Science Society, 1986. 26(2): p. 125-128.

15. McDonald, A., et al., Y-STR analysis of digital and/or penile penetration cases with no detected spermatozoa. Forensic Science International: Genetics, 2015. 15: p. 84-89.

16. Szkuta, B., K.N. Ballantyne, and R.A. van Oorschot, Transfer and persistence of DNA on the hands and the influence of activities performed. Forensic Science International: Genetics, 2017. 28: p. 10-20.

17. Szkuta, B., et al., Transfer and persistence of non-self DNA on hands over time: Using empirical data to evaluate DNA evidence given activity level propositions. Forensic Science International: Genetics, 2018. 33: p. 84-97. 
380

381

382

383

384

385

386
18. Iuvaro, A., et al., Male DNA under female fingernails after scratching: transfer and persistence evaluation by RT-PCR analysis and Y-STR typing. International journal of legal medicine, 2018. 132(6): p. 1603-1609.

19. Flanagan, N. and C. McAlister, The transfer and persistence of DNA under the fingernails following digital penetration of the vagina. Forensic Science International: Genetics, 2011. 5(5): p. 479-483. 